\title{
Multi-Organ Failure, CTCAE
}

National Cancer Institute

\section{Source}

National Cancer Institute. Multi-Organ Failure, CT CAE. NCI Thesaurus. Code C143680.

A disorder characterized by progressive deterioration of the lungs, liver, kidney and clotting mechanisms. 